\title{
Laparoscopic sleeve gastrectomy as day-case surgery: a case-matched study.
}

Lionel Rebibo $\mathrm{MD}^{1}$, Abdennaceur Dhahri MD${ }^{1}$, Rachid Badaoui $\mathrm{MD}^{2}$, Vincent Hubert $\mathrm{MD}^{2}$, Emmanuel Lorne MD, $\mathrm{PhD}^{2,3}$, Jean-Marc Regimbeau MD, $\mathrm{PhD}^{1,3}$.

1. Department of Digestive Surgery, Amiens University Hospital, Avenue René Laennec, F-80054 Amiens Cedex 01, France

2. Department of Anaesthesiology, Amiens University Hospital, Avenue René Laennec, F-80054 Amiens Cedex 01, France

3. SSPC (Simplification des Soins des Patients Complexes) - Clinical Research Unit, University of Picardie Jules Verne, 80054 Amiens Cedex 01, France

Running Title: Outpatient laparoscopic sleeve gastrectomy.

\section{Correspondence:}

Professor J.-M. Regimbeau

Service de chirurgie digestive, Hôpital Sud, CHU d'Amiens, Avenue René Laennec, F-80054 Amiens Cedex 01, France

Phone: +33 322668301

Fax: +33 322668680

E-mail: regimbeau.jean-marc@chu-amiens.fr

Word count of manuscript: 4902

Number of references: 41

Number of Figure: 2

Number of Table: 3

\section{Authors stated no financial relationship to disclose.}


Conflict of interest statements: Authors stated no conflict of interest

Abbreviations: sleeve gastrectomy (SG), gastric banding (GB), Roux-en-Y gastric bypass (RYGB), obstructive sleep apnea syndrome (OSA), day-case surgery (DCS), postoperative day (POD), body mass index (BMI), continuous positive airway pressure (CPAP). 


\section{ABSTRACT}

Background. Few series have demonstrated the feasibility of laparoscopic sleeve gastrectomy (SG) as day-case surgery (DCS).

Objective. Compare the outcomes and healthcare costs of SG performed as DCS or as an inpatient procedure.

Setting. University Hospital, France, public practice.

Methods. This was a prospective, non-randomized study of 250 consecutive patients undergoing day-case SG from May 2011 to June 2017. Each patient in the DCS group $(\mathrm{n}=250)$ was manually paired by gender, age, BMI, preoperative comorbidities and year of surgery with one patient undergoing SG as an inpatient procedure (SG control group, $\mathrm{n}=250$ ). Patients in the SG control group were excluded from DCS on the basis of DCS criteria. The primary endpoint of this study was the clinical and economic impact of performing SG as DCS compared to inpatient management. The secondary endpoints were related to DCS, DCS satisfaction rate, comparison of outcomes and costs between DCS and inpatient procedures, and the changing modalities of SG as DCS in our institution (by comparing the first 100 patients to the last 150 patients).

Results. A total of 1,573 patients underwent SG during the period: 250 patients underwent SG as DCS (15.9\%) and 554 patients were excluded on the basis of DCS criteria. No postoperative deaths, 19 overnight admissions (7.6\%), 16 unscheduled consultations $(6.4 \%)$ and 12 unscheduled hospitalizations (4.8\%) were observed in the DCS group. No significant differences were observed in postoperative complications. Readmission was higher in the DCS group (5.6\% vs. $4 \%$; $<<0.001)$, while the length of rehospitalization was shorter in the DCS group (5.8 days vs. 10.8 days; $\mathrm{p}<0.001$ ). Overall cost and cost per patient were significantly lower in the DCS group $(\mathrm{p}<0.001)$. 
25 Conclusion. Day-case SG on selected patients was not associated with increased morbidity and mortality rates and was cost-effective due to the low cost of management of postoperative complications.

Keywords: laparoscopic sleeve gastrectomy; day-case surgery; ambulatory surgery; outpatient surgery; cost analysis. 


\section{INTRODUCTION}

Day-case surgery (DCS) is a hot topic in gastrointestinal surgery. DCS was primarily validated for inguinal hernia repair, cholecystectomy $\left.{ }^{[1]}\right]$ and the treatment of gastroesophageal reflux disease ${ }^{[2,3]}$. New indications for DCS in the field of gastrointestinal surgery have been recently described, such as laparoscopic sleeve gastrectomy (SG) ${ }^{[4]}$, ileostomy closure ${ }^{[5]}$, colectomy ${ }^{[6]}$ and liver resection ${ }^{[7]}$.

In terms of bariatric procedures, many series of laparoscopic adjustable gastric banding (LAGB) performed in a day-case setting have been published ${ }^{[8,9]}$, All published series concern old series and are probably due to the decreasing rate of LAGB as treatment of

obesity ${ }^{[10]}$ these last years. In contrast, SG has become increasingly popular over recent years and now represents the leading bariatric procedure performed in USA and France ${ }^{[11,12]}$. SG is a safe procedure for the surgical treatment of obesity with a mortality rate of $0.2 \%{ }^{[13]}$. Along with postoperative hemorrhage and gastric stenosis, gastric leak (GL) is the most dreaded postoperative surgical complication of SG. According to an expert panel consensus based on experience of more than $12000 \mathrm{SG}$, the incidence of this complication is a $1.06 \%{ }^{[14]}$. Overall, $\mathrm{SG}$ is a reproducible, codified procedure with a short operating time and simple perioperative management ${ }^{[15]}$. It therefore meets the criteria for implementation of DCS. Recent published studies have demonstrated the feasibility of SG as DCS ${ }^{[4,16,17]}$, but most of these studies were based on retrospective series.

The objective of this case-matched study was to compare day-case SG and conventional hospitalization by assuming comparable results with substantial cost differences in favor of DCS.

\section{MATERIAL AND METHODS}


55 The first 100 patients were included in a prospective, non-randomized study of patients undergoing day-case SG. The study was part of a local research protocol registered as "Feasibility of Laparoscopic Sleeve Gastrectomy in Day-Case Surgery (GASTRAMBU)" (ClinicalTrials.gov identifier: NCT01513005) ${ }^{[4]}$. This study was approved by the local institutional review board (Comité de Protection des Personnes Nord Ouest II). Data concerning the other 150 patients were prospectively registered in our database. At a preoperative consultation, patients were given a study information sheet and a drug prescription for the days after surgery. The study information sheet was a document summarizing the information delivered orally. It explained the particularity of SG performed as DCS compared to conventional hospitalization (clinical pathway). All patients provided their written informed consent to participate in the study (Figure 1).

\section{Inclusion and exclusion criteria}

Inclusion criteria were as follows:

- Obesity-related criteria: body mass index (BMI) between 35 and $60 \mathrm{~kg} / \mathrm{m}^{2}$, absence of significant medical history (cardiovascular and/or pulmonary diseases, no history of major abdominal surgery) and validation by a multidisciplinary obesity staff meeting $[18]$.

- DCS criteria: treatment-compliant patients aged between 18 and 60 years, living within an hour's drive of a hospital and with an on-site support person available for the night after surgery, access to a telephone and an American Society of Anesthesiologists score of I, II or stabilized III ${ }^{[19]}$.

Exclusion criteria were as follows: 
- Obesity-related criteria: failure to meet the French national criteria for the surgical treatment of obesity, poorly controlled diabetes (because of the risk of postoperative decompensation), heart disease (history of myocardial infarction, cardiac arrhythmia, anticoagulant and/or antiplatelet therapy), BMI higher than 60 (a risk factor for complications after bariatric surgery) ${ }^{[20]}$, and obstructive sleep apnea syndrome (OSA) ${ }^{[21]}$. Our institution's obesity-related criteria have recently been modified and OSA adequately treated by continuous positive airway pressure (CPAP) no longer constitutes an exclusion criteria based on recent published series ${ }^{[17]}$ and our own experience.

- DCS criteria: under-age patient, poor treatment compliance, living more than an hour's drive from a hospital, lack of access to a telephone and the absence of an on-site carer for the night after surgery.

\section{Bariatric preoperative management}

The indication for bariatric surgery was validated in accordance with French national guidelines at a multidisciplinary obesity staff meeting ${ }^{[22]}$. Preoperative assessment was the same regardless of the type of management (DCS or inpatient). All patients attended a surgical consultation and a nutrition/dietetics consultation. They also underwent endocrine and psychological assessments. Screening for achalasia, hiatus hernia, gastritis and Helicobacter pylori infection was performed endoscopically. Helicobacter pylori infections were always eradicated prior to SG. Pulmonary function tests and overnight polysomnography were routinely performed prior to surgery in our institution to screen for OSA. 


\section{DCS preoperative management}

During the preoperative consultation, all patients received a study information sheet with the surgeon's and emergency department's telephone numbers. Patients were also instructed to accurately measure their heart rate following their discharge home after surgery. At the preoperative consultation, patients were also given a drug prescription for the days following surgery. The prescription comprised oral analgesics (acetaminophen 1 gram qid and tramadol $100 \mathrm{mg}$ tid, an antiemetic (metoclopramide $10 \mathrm{mg}$ po tid), an anticoagulant (subQ enoxaparin $40 \mathrm{IU}$ qd for 15 days), and a proton pump inhibitor (omeprazole $40 \mathrm{mg}$ po qd for three months). Patients were treated in a dedicated DCS unit. On the day of surgery, patients were admitted to the unit at 7:15 am and entered the operating theater at 8:00 am. Surgery began at around 8:30 am.

\section{Anesthesia protocol}

115 The anesthesia protocol was specifically developed for day-case $\mathrm{SG}^{[23]}$.

All patients took effervescent cimetidine $(400 \mathrm{mg})$ before leaving home for the hospital. Anxious patients were premedicated with hydroxyzine $1.5 \mathrm{mg} / \mathrm{kg} 30$ minutes prior to anesthesia.

In the operating theater, patients were pretreated with an oxygen fraction of 1 for 5 minutes. Antibiotic prophylaxis (cefazolin $2 \mathrm{~g}$ or, for patients with a history of penicillin allergy, clindamycin $600 \mathrm{mg}+$ gentamicin $3 \mathrm{mg} / \mathrm{kg}$ ) was initiated. General anesthesia was initiated by rapid sequence induction with propofol $(2.5 \mathrm{mg} / \mathrm{kg})$ and succinylcholine $(1 \mathrm{mg} / \mathrm{kg})$. 
Anesthesia was maintained with remifentanil and 4-6\% desflurane. Muscle relaxation was achieved by administration of cisatracurium $0.2 \mathrm{mg} / \mathrm{kg}$ or rocuronium $0.6 \mathrm{mg} / \mathrm{kg}$. Drug doses were based on the calculated ideal body weight. Mechanical ventilation was used to obtain an arterial oxygen saturation greater than $95 \%$ and an arterial carbon dioxide partial pressure between 30 and $35 \mathrm{mmHg}$ (with an inspiratory oxygen concentration of 50\%). In all cases, neuromuscular blockade was reversed with neostigmine bromide $40 \mu \mathrm{g} / \mathrm{kg}$ and atropine $1 \mathrm{mg}$ or sugammadex 2 to $4 \mathrm{mg} / \mathrm{kg}$ when the train-of-four ratio was higher than 2 . Postoperative analgesia was ensured by a combination of nefopam $20 \mathrm{mg}$, tramadol $100 \mathrm{mg}$ and acetaminophen $1 \mathrm{~g}$.

To prevent postoperative nausea and vomiting, patients received IV injections of (i) dexamethasone $4 \mathrm{mg}$ and droperidol $0.625 \mathrm{mg}$ on induction of anesthesia and (ii) ondansetron $4 \mathrm{mg}$ postoperatively. The nasogastric tube was always removed in the operating theater after surgery.

\section{Surgical procedure}

Laparoscopic sleeve gastrectomy was performed according to the procedure described by Dhahri et al. ${ }^{[24]}$ using the four-trocar technique, with open laparoscopy for obese patients. Infiltration of the trocars under visual control was systematically performed using $20 \mathrm{~mL}$ of ropivacaine $(7.5 \mathrm{mg} / \mathrm{mL})$. Stapling was performed using a 34-gauge French bougie. Reinforcement of the gastric staple line was not performed in the context of day-case SG. An intraoperative methylene blue test was always performed prior to the end of the procedure. Abdominal drains were never left in place (unscheduled overnight admission in such cases). 
145 Before deflation of the pneumoperitoneum, $20 \mathrm{ml}$ of ropivacaine $(2 \mathrm{mg} / \mathrm{ml})$ were instilled under the left and right diaphragmatic domes.

The surgical procedure was the same for patients undergoing SG as DCS or as an inpatient procedure.

\section{Postoperative management for DCS}

After surgery, patients were initially admitted to the recovery room and then to the DCS unit for assessment of vital signs (temperature, heart rate, blood pressure and oxygen saturation), postoperative nausea and pain (according to the classification published by Serlin et al. ${ }^{[25]}$ ) and collection of a blood sample for determination of serum hemoglobin. An upper gastrointestinal oral contrast study was performed in the first 100 cases to screen for gastric leak; patients with abnormal test results were hospitalized for observation (unscheduled overnight admission).

In the absence of any clinical, biochemical and radiological contraindications, the surgeon authorized oral refeeding (oral fluids and soft diet for the first week, then mixed diet for two weeks). The patient was deemed suitable for discharge on the same day (i) in the absence of any particular symptoms after food intake in the DCS unit and (ii) after examination by both the surgeon and the anesthetist in charge of the operation. When these conditions were not met, the patient was hospitalized overnight in the department of Digestive Surgery. Before leaving the unit, the patients were provided with dietary advice, an information sheet describing symptoms that require emergency consultation (fever, tachycardia and pain not relieved by the prescribed analgesics) and the surgeon's 24/7 telephone number. 
Patients were always contacted by the surgeon in the evening after surgery and again on POD1 by the DCS unit nurse. The patient was asked to report body temperature, heart rate and any incident abdominal pain, nausea and/or vomiting. Patients were always reviewed at follow-up visits on POD4 comprising a complete clinical assessment and laboratory tests (blood counts, C-reactive protein level, etc.). Patients were also seen in post-operative scheduled consultation at POD 30.

\section{Postoperative management for inpatients}

175 Patients undergoing SG as an inpatient procedure were admitted in the evening before surgery. Routine laboratory tests were not performed postoperatively. Oral feeding was allowed in the evening after SG or on the following day when surgery was performed in the afternoon. Discharge was authorized on the day after surgery in the absence of any particular symptoms after food intake during hospitalization (no nausea or vomiting after oral intake). Otherwise, hospitalization was prolonged for at least one day. There was no minimum value of liquid consummation to authorize discharge from hospital. Routine upper gastrointestinal oral contrast study was performed in some cases (during the same period as the first 100 cases of SG as DCS).

Patients undergoing SG as an inpatient procedure were not reviewed at follow-up visits on POD4 unlike patients managed as DCS. The post-operative scheduled consultation was performed at POD 30.

\section{Follow-up procedures}


Patients were routinely reviewed in the outpatients department after 1 month, 3 months, 6 months, and then annually. The surgeon determined the level of satisfaction with day-case SG three months after SG.

\section{Case matching procedure}

Each patient undergoing SG as DCS (DCS group) was manually paired by gender, age, BMI 195 and preoperative comorbidities (hypertension, diabetes mellitus, dyslipidemia, metabolic syndrome (as defined by the National Cholesterol Education Program (Adult Treatment Panel III) guidelines (NCEP-ATP III), NCEP-ATP3) and OSA) and year of surgery (relative to the routine upper gastrointestinal oral contrast study) with one patient undergoing SG as an inpatient procedure (control group). Case-matched analysis was performed by selecting patients for the control group from the group of patients excluded from DCS on the basis of the DCS criteria ( $\mathrm{n}=554$ patients). For the last patient with OSA included in the DCS group, a case-matched study was performed on the OSA population treated by CPAP.

The case-matching procedure between each SG performed as DCS (DCS group) and each control patient (control group) was performed without knowledge of the outcomes after 205 surgical procedure in the two groups. This case-matching procedure was used in order to avoid bias that would otherwise have influenced our results.

\section{Economic assessment}

All direct costs were calculated on an individual basis. Economic assessment was performed according to French guidelines evaluating direct medical costs over a 3-month period starting from the SG procedure. Direct medical costs were evaluated from a societal perspective (national health insurance). Indirect costs were not calculated as part of the study design 
because of the lack of comprehensive data. Costs were not discounted and were expressed in Euros.

215 Data included hospitalization and rehospitalization costs (according to the CCAM and GHM codes transmitted for hospital funding) and were extracted from the patient's medical records. Scheduled consultation were included in hospitalization cost. Scheduled consultation on POD4 (with inclusion of laboratory assessment) were also included specifically on hospitalization cost for patients undergoing SG as DCS. The sources of funding were the manufacturer's prices and list of benefits (LPP) and the national economic scale (ENC). Costs were calculated for each individual patient on the basis of the inclusion year.

Hospitalization costs included operating room use costs (laparoscopy device, staple device), anesthesiology costs, and surgical instrumentation, postoperative care, and length of hospital stay. For patients undergoing SG as conventional hospitalization, costs of hospitalization were taken into account using two different methods: cost of hospitalization without taking into account the admission the day before surgery (cost analysis starting the day of the surgery) and cost analysis taking into account the cost of admission the day before surgery. Rehospitalization costs included length of hospital stay (additional costs for ICU hospitalization), postoperative care, reoperation and/or radiological and/or endoscopic procedures. Overall cost was represented by hospital stay cost during primary SG and rehospitalization cost in the case of postoperative complications during the first 3 postoperative months for all of the 250 patients. Mean overall cost per patient represents the mean cost per patient including the cost of the hospital stay during primary SG and rehospitalization costs in the event of postoperative complications during the first 3 postoperative months. 


\section{Study endpoints}

The primary endpoint of the study was the clinical and economic impact of performing SG as DCS compared to inpatient management. The secondary endpoints were variously related to DCS (unscheduled overnight admission rate, unscheduled consultation rate, hospital readmission rate, reoperation rate, complication rate (frequency of complications during and after surgery according to the Clavien classification [26], and the frequency of major complications (with a Clavien score $\geq 3$ )), level of patient satisfaction with day-case SG at 3 months, comparison of outcomes and cost between DCS and inpatient management, and the changing modalities of SG as DCS in our institution.

\section{Statistical analysis}

Between-group comparisons of quantitative variables were performed with a t-test for paired series, whereas between-group comparisons of qualitative variables (including the primary endpoint) were performed with a McNemar's Chi-square test. Results are expressed as mean \pm standard deviation and range (for quantitative variables) or number and percentage (for qualitative variables). The limit of statistical significance was $\mathrm{p} \leq 0.05$. All statistical analyses were performed with SAS software (version 9.2, SAS Institute, Cary, NC, USA).

\section{RESULTS}

\section{Study population}

During the study period, 1,573 patients underwent SG (with or without previous gastric banding). Two hundred fifty patients from this population agreed to undergo day-case LSG 
(15.9\%). The reasons for exclusion from DCS for the remaining 1,573 patients were as follows: criteria for DCS in 554 cases, OSA in 482 cases (including 381 cases treated by CPAP), previous gastric banding in 120 cases, significant medical history in 78 cases, $\mathrm{BMI} \geq$ $60 \mathrm{~kg} / \mathrm{m}^{2}$ in 47 cases and poorly controlled diabetes in 42 cases (Figure 2). Finally, after excluding patients with a history of gastric banding, the inclusion rate for DCS was $17.2 \%$.

The group of 250 patients consisted of 232 women (92.8\%) with a mean (range) age of 35.4 years $\pm 7.7(18-58)$ and a mean BMI of $42.2 \mathrm{~kg} / \mathrm{m}^{2} \pm 2.9(35-52.3)$. Nine patients had a BMI $\geq$ $50 \mathrm{~kg} / \mathrm{m}^{2}(3.6 \%)$. Observed comorbidities included hypertension $(17.2 \%, \mathrm{n}=43)$, controlled type II diabetes $(4 \%, \mathrm{n}=10)$, dyslipidemia $(9.2 \%, \mathrm{n}=23)$ and metabolic syndrome $(2 \%, \mathrm{n}=$ 5). Six patients had OSA treated by CPAP therapy (Table 1).

\section{Operative data}

Only one SG was generally performed on each list. However, for 60 patients, two SG procedures were performed successively on the same day. All procedures were performed laparoscopically. The mean \pm SD (range) operating time was 54 minutes \pm 14.5 (30-105)

(Table 1). One patient required introduction of an additional trocar (due to difficult exposure) and 21 patients required unique suture for arteriolar bleeding on the gastric staple line $(8.4 \%)$. One patient had the gastric fundus impacted into the spleen, in whom a part of gastric serosa of the fundus was left attached to the splenic capsule. No gastric leak was diagnosed on the intraoperative methylene blue test and no drainage of the abdominal cavity was performed. One patient developed erythema in the absence of hypotension upon anesthetic induction, but this reaction did not affect the surgical procedure. Orotracheal intubation was difficult in two other patients. 


\section{Day of surgery}

All patients were admitted to the recovery room after surgery. The mean (range) length of stay in the recovery room was 70 minutes (35-240). The mean (range) vital signs and laboratory test values on admission to the DCS unit were as follows: heart rate: $79 \mathrm{bpm}$ (55118); arterial blood pressure: $137 / 80 \mathrm{mmHg}$ (105/65-178/90); body temperature: $36.5^{\circ} \mathrm{C}(36-$ 37.5); hemoglobin level: $14.1 \mathrm{~g} / \mathrm{dl}$ (11.3-16.9); white blood cell count: 16,623 (8,600-20,400). The first 100 patients of our series underwent upper gastrointestinal oral contrast study, which did not reveal any abnormalities (including signs of gastric fistula). An upper gastrointestinal oral contrast study was not performed in the remaining 150 patients.

Oral feeding was resumed an average of 415 minutes \pm 45 (300-585) after admission to the DCS unit. Two hundred thirty-one patients were discharged in the evening after surgery, after a mean length of stay in the DCS unit of 615 minutes \pm 30 (575-675).

\section{Criteria related to day-case surgery}

No postoperative deaths were observed. There were 19 unscheduled overnight admissions due to pain in 8 cases, discomfort after performing the UGI study in three cases, somnolence in the day-case unit in two cases, difficulty of intubation during surgery in two cases, nausea in two cases, gastric fundus impacted into the spleen in one case and anxious patient in one case.

There were 16 unscheduled consultations $(6.4 \%)$ by 15 different patients $(6.0 \%)$. Nine of these unscheduled consultations resulted in hospitalization $(56.2 \%$ of unscheduled consultations). There were 12 unscheduled hospitalizations, including three patients 
hospitalized after a scheduled consultation. Reasons for hospitalization were as follows: four cases of gastric leak, one case of gastric stricture, one case of pneumonia, two cases of perigastric hematoma requiring radiological drainage in one case, one case of abdominal pain secondary to ischemia of the upper pole of the spleen requiring morphine analgesia for two days, one case of wound hematoma secondary to thrombophilia, and two cases of scapular pain related to laparoscopy.

\section{Consultation on postoperative day 4}

Two hundred forty-eight of the 250 patients were examined on POD4. Two patients were hospitalized on POD4, one for scapular pain and one for wound hematoma. Two hundred forty-three of the 248 patients did not report any specific somatic complaints.

315 During the scheduled consultation on POD4, seven patients with high CRP levels $(\geq 120$ $\mathrm{mg} / \mathrm{dl}$ ) were assessed by oral contrast-enhanced abdominal computed tomography (CT) scan. Four of these seven patients had no leak and one patient presented signs of ischemia of the upper pole of the spleen with no clinical symptoms. GL was diagnosed on abdominal CT scan in the remaining three patients.

\section{Complications}

\section{$\underline{\text { Major complications }}$}

Six major complications (Clavien score $\geq 3$ ) were recorded $(2.4 \%)$, including four cases of gastric leak, one case of postoperative hematoma and one case of gastric stricture. In three patients, gastric leak was diagnosed during the scheduled consultation on POD4. One of these 
three patients had no clinical symptoms and the GL was diagnosed in the presence of elevated CRP. These three patients underwent laparoscopic exploration with peritoneal lavage and an intraoperative methylene blue test, which revealed a leak at the upper edge of the staple line, which was then sutured, placement of a drainage tube and placement of a feeding jejunostomy. Seven days after reoperation, two double pigtail stents were inserted and the patients were discharged from hospital a median of 15 days (14-16) after reoperation. In the other patient, GL was diagnosed on POD 37. This patient underwent first-line endoscopic drainage using two double pigtail stents. The length of hospital stay was 3 days. Six weeks after double pigtail stenting, CT scan was performed to demonstrate closure of the gastric leak and the double pigtail stents were removed on the day after CT scan for all patients.

One patient was reviewed on POD 7 for abdominal pain in the absence of fever. Abdominal CT scan revealed an intra-abdominal hematoma. This patient underwent radiological drainage and was discharged five days later. The patient with gastric stricture underwent three pneumatic dilations, which were effective, but required total parenteral nutritional support for 340 four days.

\section{$\underline{\text { Minor complications }}$}

Three minor complications with a Clavien score of two were observed: ischemia of the upper pole of the spleen $(n=1)$, perigastric hematoma $(n=1)$ and lung infection $(n=1)$. Five patients 345 also presented a Clavien score of one with scapular pain $(n=2)$, poor wound closure $(n=1)$, recurrent vomiting $(n=1)$ and wound hematoma secondary to thrombophilia $(n=1)$.

\section{$\underline{\text { Patient satisfaction and compliance with day-case SG }}$}


The patient satisfaction rate evaluated at the third postoperative month after day-case SG was 96.8\%. The eight dissatisfied patients were those who experienced a gastric leak $(n=4)$, scapular pain $(n=2)$ and gastric stenosis $(n=1)$. The overall compliance with our protocol was $85.2 \%$ (213 out of 250 patients)

\section{Case-matched analysis}

355 Comparison of intraoperative data revealed a significant difference in terms of a decreased operating time in the DCS group $(\mathrm{p}<0.001)$. Comparison of postoperative data revealed a significant difference in terms of mean length of hospital stay (0.1 days vs. 1.9 days; $\mathrm{p}<0.001)$. The readmission rate was higher in the DCS group (5.6\% vs. $4 \%$; $\mathrm{p}<0.001)$, while the length of rehospitalization was shorter in the DCS group (5.8 days vs. 10.8 days; $\mathrm{p}<0.001)$. No significant difference was observed in terms of postoperative complications (Table 1).

\section{Economic assessment}

SG performed as DCS was significantly cheaper than inpatient SG whether or not an unplanned admission was required. There was a reduction of cost of initial surgery when SG was performed as DCS ranging from 19\% (including overnight admission; $\mathrm{p}=0.03$ ) to $43 \%$ (excluding overnight admission; $\mathrm{p}<0.001)$. The mean cost of rehospitalization $(€ 7,314$ vs. $€ 7,559 ; \mathrm{p}=0.86)$ and the median cost of GL (€7,950 vs. $€ 8,630 ; \mathrm{p}=0.41)$ were similar between the DCS and inpatient groups. In terms of the overall cost of SG and the overall cost per patient, DCS was significantly more cost-effective than inpatient management, regardless of the postoperative course (Table 2). The overall cost per patient if SG was performed as 
DCS was reduced from $36 \%$ to $42 \%$ compared to conventional hospitalization depending on whether there is or not unplanned overnight admission and/or rehospitalisation $(\mathrm{p}<0.001)$. When taking into account the cost of admission the day before surgery, differences were even

\section{Changing modalities of SG as DCS in our institution}

Comparison of the first 100 cases and the subsequent 150 cases of our experience demonstrated similar demographic data except for OSA patients, as patients with OSA adequately treated by CPAP were progressively included in the DCS procedure (0\% vs. 6\%; $\mathrm{p}=0.04$ ). Unplanned overnight admission rates and unscheduled consultation rates were similar despite changes in DCS criteria. The only observed changes concerned the readmission rate, which was lower over the second period ( $7 \%$ vs. $4.6 \%$; $=0.03$ ). Analysis of postoperative complications showed a significantly lower GL rate (3\% vs. $0.6 \% ; \mathrm{p}=0.01$ ), partly explaining the significantly lower reoperation rate $(1.2 \%$ vs. $0 \% ; \mathrm{p}=0.03)$.

\section{DISCUSSION}

Day-case surgery has become increasingly popular over recent years and new innovative DCS procedures have been described ${ }^{[5-7,27]}$. In parallel, bariatric surgery rates are exploding worldwide due to the good results of this surgery in terms of weight loss, correction of cardiovascular risk factors and change in lifestyle ${ }^{[28]}$. Bariatric surgery as DCS is feasible and has already been described for gastric banding ${ }^{[\mathbf{8}, 29]}$, but all of these series were published several years ago and the GB rate has decreased over recent years ${ }^{[30]}$. Four series of bariatric surgery as DCS have been recently published (three on $\mathrm{SG}^{[4,16,17]}$ and one on RYGB ${ }^{[31]}$ ). 
The problem with published series is that most of them are retrospective studies and only two of these studies defined inclusion and exclusion criteria for DCS ${ }^{[4,17]}$, which prevents other centers from performing this type of outpatient procedure. Case series are necessary to demonstrate the possibility of performing these types of procedures as DCS. Morton et al ${ }^{[31]}$ reported on a large prospective clinical database of patients undergoing RYGB, including 507 patients as DCS. In their series, patients with DCS had a higher 30-day readmission rate and a 13-fold increased risk of death $(0.8 \%)$ compared to the reference length of stay of two days. The problem with these series is the number of patients undergoing RYGB as DCS $(n=507)$ and that the number of centers performing these procedures as DCS is not specified. Inclusion and exclusion criteria are also lacking. This type of series perfectly illustrates that ambulatory bariatric surgery must be rigorously monitored to avoid increased morbidity and mortality. RYGB can probably be performed as DCS, but more rigorous criteria are required for this type of management.

Our early experience based on our first 100 cases has been published previously. The present series shows our changing management based on our previous experience. For example, we routinely performed upper gastrointestinal (UGI) oral contrast studies to screen for GL during the first 100 cases, in order to increase the safety of SG, although some series have shown that routine UGI contrast studies on POD one or two ${ }^{[32-34]}$ are not useful for the detection of GL. In our previous series, upper gastrointestinal oral contrast studies were responsible for $50 \%$ of our unplanned overnight admissions. As UGI contrast study is not useful for the detection of 415 GL and is not cost-effective ${ }^{[35]}$, we subsequently decided to stop performing this assessment. Our exclusion criteria have also changed over time. In our previous series, patients with OSA, regardless of the grade, were excluded from DCS. Garofalo et al ${ }^{[17]}$ includes patients with mild, moderate or severe OSA treated with CPAP. Based on their experience, a retrospective 
analysis of a large American database ${ }^{[36]}$ and a retrospective review of our database of patients with OSA, patients with OSA adequately treated by CPAP are no longer excluded, as these patients do not present an increased risk of postoperative complications after SG.

No published series have compared DCS to inpatient SG based on the same preoperative demographic data. For this reason, we performed a case-matched study by selecting patients in our inpatient group that could underwent DCS but were excluded because of social criteria of DCS. This selected population have no major other comorbidities (such as asthma, chronic obstructive pulmonary disease or others) in order to avoid possible bias in our results. The major finding of our case-matched study is that DCS is not associated with an increased risk of morbidity, as the overall complication rates were similar in the two groups and the major complication rate was lower in the DCS group (2.4\% vs. $3.6 \%$; $=0.68)$. The readmission rate was higher in the DCS group $(\mathrm{p}<0.001)$, probably due to our policy of keeping the patient in hospital after an unscheduled consultation following day-case surgery. Some complications, such as shoulder pain, were also observed in the DCS group and required rehospitalization, while these complications were not observed in patients of the control group, who were already hospitalized and only required longer hospitalization. For this reason, the mean length of rehospitalization was shorter after DCS compared to inpatient management (5.8 days vs. 10.8 days, respectively: $\mathrm{p}<0.001)$. On the other hand, operative time was increased in case of inpatient management compared to DCS (64.2 min vs. 54 min, respectively; $p<0.001$ ) while patients are similar in the two groups. This could be explain by the fact that during SG as DCS, a nurse assists us during the surgical procedure, thus saving time in the preparation of the surgical equipment and thus making it possible to gain time on different operative times of sleeve gastrectomy. When we performed SG as inpatient, no nurse assists us during the SG explaining increased operative time. 
The GL rate in our previous series was relatively high at 3\%. Although the results of this series were similar to those reported in the literature with a mean rate of $2.3 \%$ [37], we were not satisfied with these preliminary results and decided to improve our information program for SG and DCS in particular. Our information program insisted in particular on the dietary measures allowing limiting the increase of intra-gastric pressure, major source of GL. This information was provided during individual consultation and group workshops with patients already had been operated. This new approach, with no change in the surgical technique, allowed the GL rate to be decreased to $0.6 \%(\mathrm{p}=0.03)$ (Table 3). Another important point in our series is that patients with postoperative GL following SG as DCS did not present an increased risk of complications related to DCS. The duration of GL management was similar in the DCS group and the control group, and no patients with GL were admitted to ICU for management of GL in the DCS group.

455 The main early complication after SG that could constitute a brake to SG as DCS is probably the higher postoperative bleeding rate rather than GL. Most cases of GL occur a certain time after SG, which is why patients were routinely reviewed at a POD4 consultation, based on our experience of GL, in which one-half of cases of GL occurred a median interval of 6 days after $\mathrm{SG}^{[38]}$. In our protocol, a blood sample is routinely taken for determination of hemoglobin several hours after SG. Hemoglobin assay may appear to be of limited value, as bleeding patients generally present clinical signs before a fall in hemoglobin. As no cases of bleeding requiring reoperation were observed in our series, the real value of hemoglobin assay to screen for bleeding cannot be determined. Nevertheless, we continue to perform hemoglobin assay as a reference for the scheduled consultation at POD4. The routine use of staple line reinforcement for SG as DCS could be useful in order to further improve the safety of 
outpatient management, as most published series report a greater impact of staple line reinforcement on postoperative bleeding ${ }^{[39]}$.

This series is unusual in that it included a cost analysis, which showed that DCS was costeffective compared to conventional hospitalization. This result was expected and many series have already shown the cost-effectiveness of DCS. Only one series concerning major abdominal surgery has demonstrated the impact of DCS for laparoscopic fundoplication ${ }^{[40]}$. The benefit of DCS compared to conventional hospitalization is almost doubled in the absence of unplanned overnight admission $(€ 2,944$ vs. $€ 5,151 ; \mathrm{p}<0.001)$ and the significantly shorter length of stay in the case of an unplanned overnight admission (Table 2). Similar results were observed when analyzing the cost of rehospitalization and management of GL in particular, suggesting that DCS is not associated with poorer outcomes in the event of complications and, despite day-case management, management of complications is not delayed compared to conventional management. Our cost-analysis was also in favor of DCS regardless of the outcomes of SG as DCS (with or without readmission) (Table 2), which could be due to our DCS inclusion criteria that ensure a low complication rate, and standardization of the management of postoperative complications in our center ${ }^{[41]}$ allowing rapid discharge with no increased morbidity so that complications have a minimal impact on the overall cost of management. In our cost analysis, if we also analyse cost after including cost of admission the day before surgery, the difference is even more significant (Table 2).

This study presents several limitations. Firstly, the unscheduled consultation rate for the control group was not available, preventing any comparison between the DCS and control groups. Secondly, the time to return to work was also not evaluated in this study. Most published series of DCS highlight the earlier return to work following DCS, but no data are currently available concerning SG as DCS. Thirdly, even if we are the only university hospital 
in our region and usually managed most of post-operative complications from different centre in our region, we cannot assure in $100 \%$ of cases that we were able to capture readmissions/rehospitalizations if patient went to outside hospitals or other centres, particularly in patients undergoing SG as inpatient. Fourth, the protocol surveillance of SG as DCS compared to conventional hospitalization is quite different, in particular concerning the scheduled consultation at POD 4 (not present in case of conventional hospitalization). At the time we started to perform GS as DCS, no series exist concerning outcomes of SG as DCS and we wanted to limit possible risk associated with DCS with such surgical procedure. However, this scheduled consultation does not impact the occurrence or not of a complications as surgical technique were similar between both groups. Based on the results of our series, we probably drop routine scheduled consultation at POD 4 as finally its impact is low. Finally, more prospective series are necessary to confirm these results and to demonstrate that day-case SG is feasible without increasing the risk of postoperative complications in selected patients, allowing day-case SG to be proposed as alternative management to inpatient surgery for selected patients in order to reduce health spending and maintain a balance between the pursuit of cost reduction and patient safety.

\section{CONCLUSION}

Day-case SG on strictly selected patients, was not associated with elevated morbidity and mortality rates. Satisfaction rates were satisfactory with an acceptable readmission rate with no increase in the severity of complications compared to conventional management. On cost analysis, SG as DCS is cost-effective, in view of the low cost of management of postoperative complications, which is not more difficult than that of conventional SG. 


\section{REFERENCES}

515 [1] Mjåland O, Raeder J, Aasboe V, Trondsen E, Buanes T. Outpatient laparoscopic cholecystectomy. Br J Surg. 1997;84:958-61.

[2] Trondsen E, Mjâland O, Raeder J, Buanes T. Day-case laparoscopic fundoplication for gastro-oesophageal reflux disease. Br J Surg. 2000;87:1708-11.

[3] Mariette C, Piessen G, Balon JM, Guidat A, Lebuffe G, Triboulet JP. The safety of the same-day discharge for selected patients after laparoscopic fundoplication: a prospective cohort study. Am J Surg. 2007;194:279-82.

[4] Rebibo L, Dhahri A, Badaoui R, Dupont H, Regimbeau JM. Laparoscopic sleeve gastrectomy as day-case surgery (without overnight hospitalization). Surg Obes Relat Dis. $2015 ; 11: 335-42$.

525 [5] Bhalla A, Peacock O, Tierney GM et al. Day-case closure of ileostomy: feasible, safe and efficient. Colorectal Dis. 2015;17:820-3.

[6] Gignoux B, Pasquer A, Vulliez A, Lanz T. Outpatient colectomy within an enhanced recovery program. J Visc Surg. 2015;152:11-5.

[7] Gaillard M, Tranchart H, Lainas P, Tzanis D, Franco D, Dagher I. Ambulatory 530 laparoscopic minor hepatic surgery: Retrospective observational study. J Visc Surg. 2015; $152: 292-6$.

[8] Kormanova K, Fried M, Hainer V, Kunesova M. Is laparoscopic adjustable gastric banding a day surgery procedure? Obes Surg. 2004;14:1237-40. 
[9] Watkins BM, Montgomery KF, Ahroni JH, Erlitz MD, Abrams RE, Scurlock JE.

Adjustable gastric banding in an ambulatory surgery center. Obes Surg. 2005; 15:1045-9.

[10] Angrisani L, Santonicola A, Iovino P, Formisano G, Buchwald H, Scopinaro N. Bariatric Surgery Worldwide 2013. Obes Surg. 2015;25:1822-32.

[11] Spaniolas K, Kasten KR, Brinkley J et al. The Changing Bariatric Surgery Landscape in the USA. Obes Surg. 2015;25:1544-6. trends in France: 2005-2011. Surg Obes Relat Dis. 2014;10:328-34.

[13] Zellmer JD, Mathiason MA, Kallies KJ, Kothari SN. Is laparoscopic sleeve gastrectomy a lower risk bariatric procedure compared with laparoscopic Roux-en-Y gastric bypass? A meta-analysis. Am J Surg. 2014;208:903-10;discussion 909-10.

545 [14] Rosenthal RJ; International Sleeve Gastrectomy Expert Panel, Diaz AA et al. International Sleeve Gastrectomy Expert Panel Consensus Statement: best practice guidelines based on experience of $>12,000$ cases. Surg Obes Relat Dis. 2012; 8:8-19.

[15] Rebibo L, Blot C, Verhaeghe P, Cosse C, Dhahri A, Regimbeau JM. Effect of perioperative management on short-term outcomes after sleeve gastrectomy: a 600-patient 550 single-center cohort study. Surg Obes Relat Dis. 2014;10:853-8.

[16] Billing PS, Crouthamel MR, Oling S, Landerholm RW. Outpatient laparoscopic sleeve gastrectomy in a free-standing ambulatory surgery center: first 250 cases. Surg Obes Relat Dis. 2014;10:101-5.

[17] Garofalo F, Denis R, Abouzahr O, Garneau P, Pescarus R, Atlas H. Fully Ambulatory 555 Laparoscopic Sleeve Gastrectomy: 328 Consecutive Patients in a Single Tertiary Bariatric Center. Obes Surg. 2016;26:1429-35. 
[18] Rebibo L, Maréchal V, De Lameth I et al. Compliance with a multidisciplinary team meeting's decision prior to bariatric surgery protects against major postoperative complications. Surg Obes Relat Dis. 2017;13:1537-1543.

560 [19] Kraft K, Mariette C, Sauvanet A et al. Indications for ambulatory gastrointestinal and endocrine surgery in adults. J Visc Surg. 2011; 148:69-74.

[20] Fernandez AZ Jr, Demaria EJ, Tichansky DS et al. Multivariate analysis of risk factors for death following gastric bypass for treatment of morbid obesity. Ann Surg. 2004;239:698702; discussion 702-3.

565 [21] Fernandez AZ Jr, DeMaria EJ, Tichansky DS et al. Experience with over 3,000 open and laparoscopic bariatric procedures: multivariate analysis of factors related to leak and resultant mortality. Surg Endosc. 2004;18:193-7.

[22] Gastrectomie Longitudinale [sleeve gastrectomy] pour obésité. Hautes autorité de la santé. Recommandations 2008.

570 [23] Badaoui R, Alami Chentoufi Y, Hchikat A et al. Outpatient laparoscopic sleeve gastrectomy: first 100 cases. J Clin Anesth. 2016; 34:85-90.

[24] Dhahri A, Verhaeghe P, Hajji H, Fuks D, Badaoui R, Deguines JB, Regimbeau JM. Sleeve gastrectomy: technique and results. J Visc Surg. 2010;147(5 Suppl):e39-46.

[25] Serlin RC, Mendoza TR, Nakamura Y, Edwards KR, Cleeland CS. When is cancer pain 575 mild, moderate or severe? Grading pain severity by its interference with function. Pain. $1995 ; 61: 277-84$ 
[26] Dindo D, Demartines N, Clavien PA. Classification of surgical complications: a new proposal with evaluation in a cohort of 6336 patients and results of a survey. Ann Surg $2004 ; 240: 205-13$

[27] Mari FS, Gasparrini M, Cosenza UM et al. Feasibility and safety study of day-case Transtar $^{\mathrm{TM}}$ procedure. Surgeon. 2013;11 Suppl 1:S6-9.

[28] Sjöström L, Lindroos AK, Peltonen M et al. Lifestyle, diabetes, and cardiovascular risk factors 10 years after bariatric surgery. N Engl J Med. 2004;351:2683-93.

[29] De Waele B, Lauwers M, Van Nieuwenhove Y, Delvaux G. Outpatient laparoscopic gastric banding: initial experience. Obes Surg. 2004;14:1108-10.

[30] Spaniolas K, Kasten KR, Brinkley J, Sippey ME, Mozer A, Chapman WH, Pories WJ. The Changing Bariatric Surgery Landscape in the USA. Obes Surg. 2015;25:1544-6.

[31] Morton JM, Winegar D, Blackstone R, Wolfe B. Is ambulatory laparoscopic Roux-en-Y gastric bypass associated with higher adverse events? Ann Surg. 2014; 259:286-92. swallow studies after laparoscopic sleeve gastrectomy are unnecessary. Am J Surg. 2014;207:897-901.

[33] Mizrahi I, Tabak A, Grinbaum R et al. The utility of routine postoperative upper gastrointestinal swallow studies following laparoscopic sleeve gastrectomy. Obes Surg. 2014;24:1415-9. 
[34] Wahby M, Salama AF, Elezaby AF et al. Is routine postoperative gastrografin study needed after laparoscopic sleeve gastrectomy? Experience of 712 cases. Obes Surg. 2013;23:1711-7.

[35] Rebibo L, Cosse C, Robert B et al. Eliminating routine upper gastrointestinal contrast 600 studies after sleeve gastrectomy decreases length of stay and hospitalization costs. Surg Obes Relat Dis. 2017;13:553-559.

[36] Berger ER, Clements RH, Morton JM et al. The Impact of Different Surgical Techniques on Outcomes in Laparoscopic Sleeve Gastrectomies: The First Report from the Metabolic and Bariatric Surgery Accreditation and Quality Improvement Program (MBSAQIP). Ann Surg. 2016;264:464-73.

[37] Zellmer JD, Mathiason MA, Kallies KJ, Kothari SN. Is laparoscopic sleeve gastrectomy a lower risk bariatric procedure compared with laparoscopic Roux-en-Y gastric bypass? A meta-analysis. Am J Surg. 2014;208:903-10;discussion 909-10.

[38] Pequignot A, Fuks D, Verhaeghe $\mathrm{P}$ et al. Is there a place for pigtail drains in the management of gastric leaks after laparoscopic sleeve gastrectomy? Obes Surg. 2012; 22:71220.

[39] D'Ugo S, Gentileschi P, Benavoli D et al. Comparative use of different techniques for leak and bleeding prevention during laparoscopic sleeve gastrectomy: a multicenter study. Surg Obes Relat Dis. 2014;10:450-4.

615 [40] Gronnier C, Desbeaux A, Piessen G et al. Day-case versus inpatient laparoscopic fundoplication: outcomes, quality of life and cost-analysis. Surg Endosc. 2014;28:2159-66. 
[41] Rebibo L, Bartoli E, Dhahri A et al. Persistent gastric fistula after sleeve gastrectomy: an analysis of the time between discovery and reoperation. Surg Obes Relat Dis. 2016;12:84-93. 


\section{FIGURE LEGENDS}

Figure 1: Study synopsis

Figure 2: Proportion of SG as DCS and reasons for exclusion from DCS during the study 625 period. 
Surgical consultation

Inclusion criteria for day-case $\mathrm{SG}$

Patient's study information

Drugs prescription

Yes

Anesthetic visit

Inclusion criteria for day-case SG
No

No

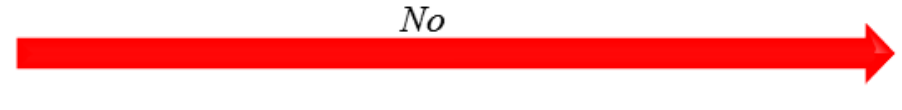

Exclusion criteria for DCS

DCS criteria
Exclusion criteria for DCS

Obesity-related criteria DCS criteria

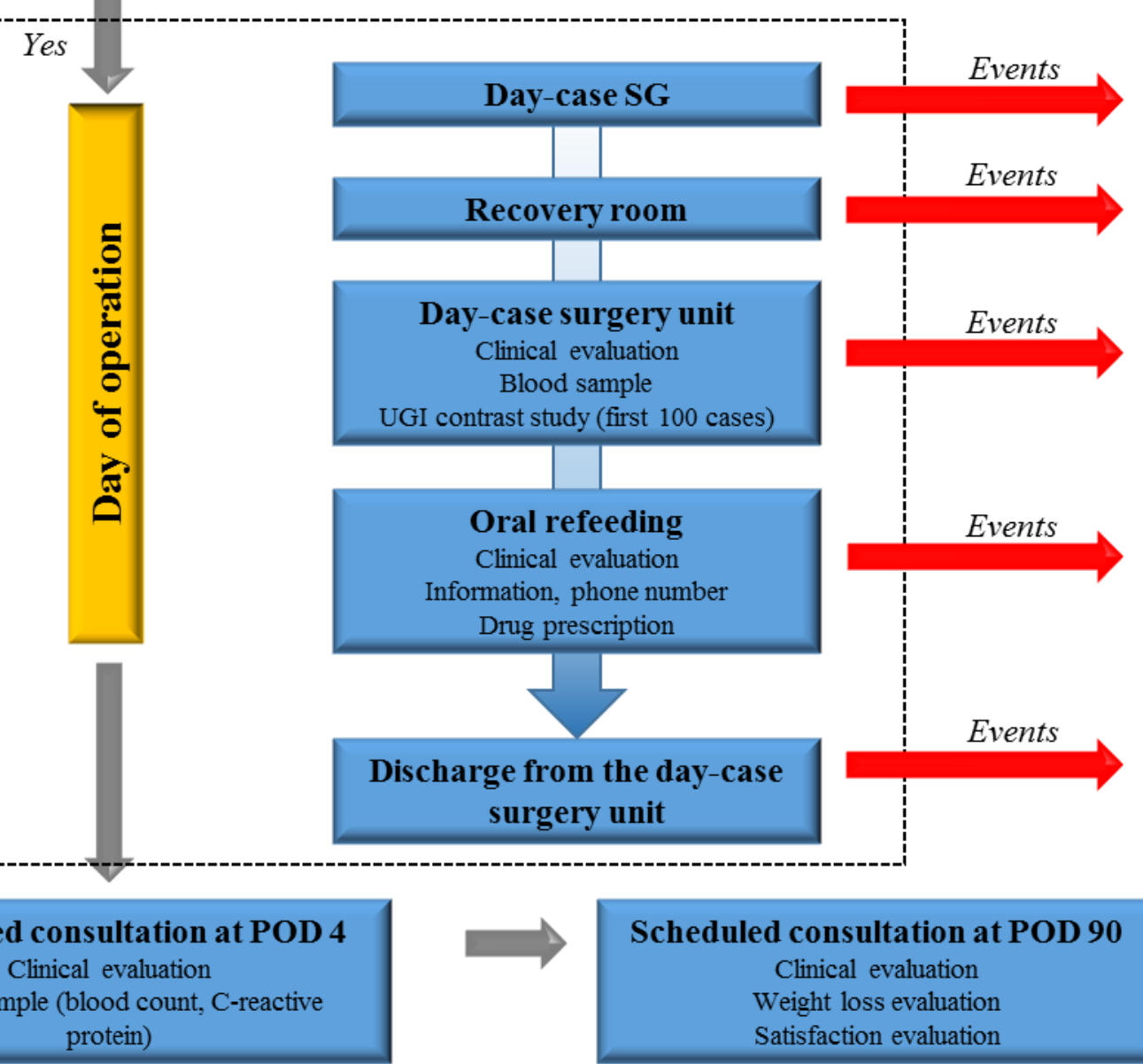




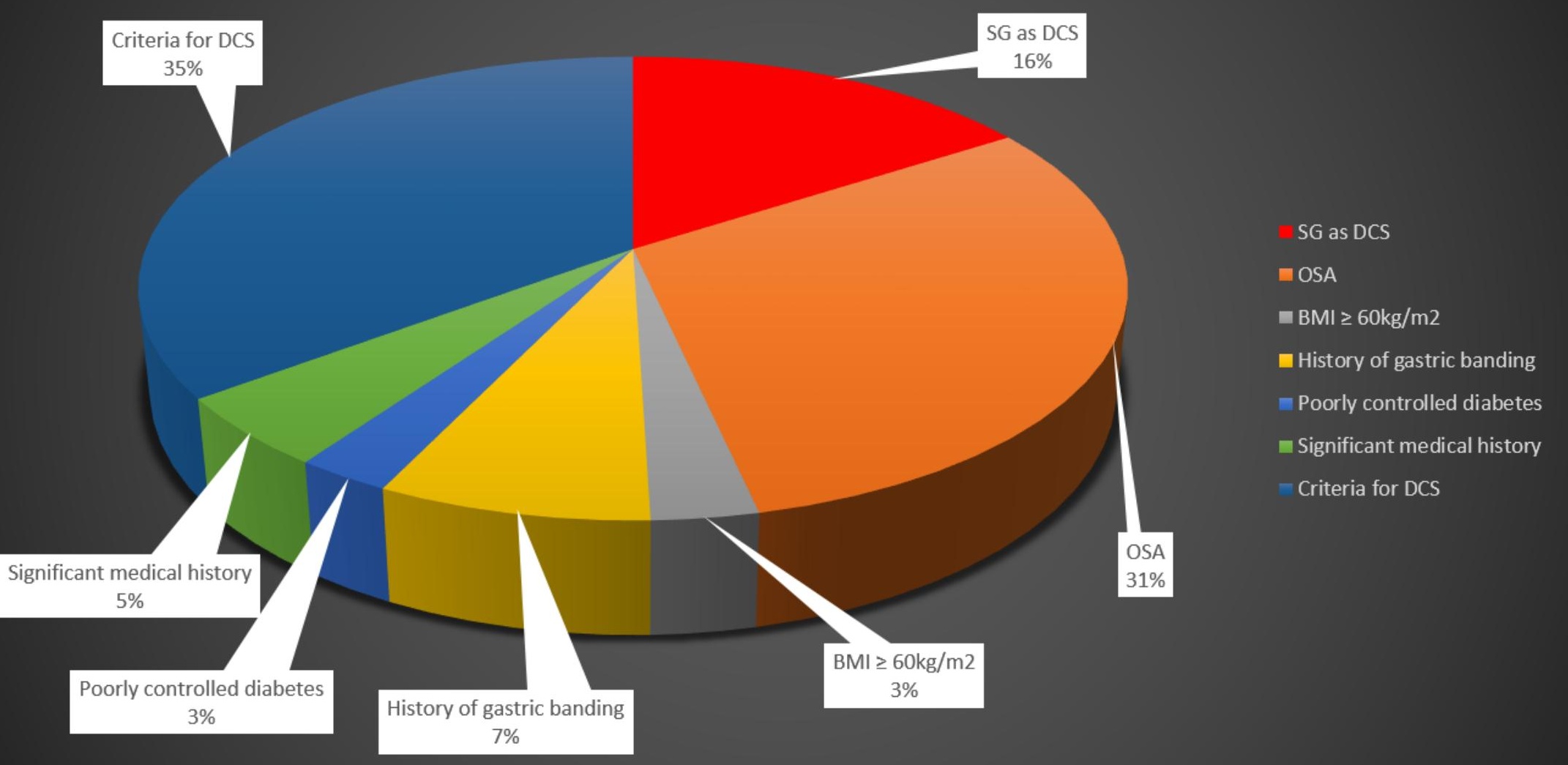


Table 1: Case-matched analysis between the DCS and control groups.

\section{DCS group Control group}

$\mathrm{n}=250 \quad \mathrm{n}=250$

\section{Preoperative data}

Age (range)

Female gender $(\%)$

BMI (range)

$\mathrm{BMI} \geq 50 \mathrm{~kg} / \mathrm{m} 2(\%)$

Type 2 Diabetes $(\%)$

Hypertension (\%)

Dyslipidemia (\%)

Metabolic Syndrome (\%)

OSA (\%)

$\begin{array}{ccc}35.3(18-58) & 35.1(18-58) & 0.83 \\ 232(93) & 232(93) & 0.86 \\ 42.2(35-52.3) & 42.3(35.3-53.9) & 0.85 \\ 10(4) & 11(4.4) & 0.66 \\ 10(4) & 10(4) & 0.99 \\ 43(17.2) & 43(17.2) & 0.98 \\ 23(9.2) & 17(6.8) & 0.32 \\ 5(2) & 6(2.4) & 0.76 \\ 6(2.4) & 6(2.4) & 0.99\end{array}$

\section{Intraoperative data}

Operating time in minutes (range)

$54(30-125) \quad 64.2(30-150) \quad<0.001$

Intraoperative events

Leak on intraoperative methylene
blue test $(\%)$
Suture of gastric staple line (\%)
Abdominal drainage $(\%)$

$0(0) \quad 0(0)$

$21(8.4)$

30 (12)

0.16

$0(0)$

$3(1.2)$

0.11

\section{Postoperative data}

Mean length of hospital stay (range)

Postoperative mortality (\%)

$0.1(0-3)$

$1.9(1-9)$

$<0.001$

$0(0)$

$0(0)$

Postoperative complications (\%)

$14(5.6)$

$15(6)$

0.69

Major postoperative complications (\%)

$6(2.4)$

9 (3.6)

0.68

Reoperation (\%)

$3(1.2)$

$6(2.4)$

0.31

Type of complications
Gastric leak (\%)
Postoperative bleeding (\%)
Postoperative hematoma (\%)
Gastric stenosis (\%)

Rehospitalization (\%)

Mean length of rehospitalization (range)

$$
4 \text { (1.6) }
$$

$0(0)$

$2(0.8)$

$1(0.4)$

$14(5.6)$

$5.8(2-15)$
5 (2)

0.74

$2(0.8)$

0.16

$2(0.8)$

0.99

$1(0.4)$

0.99

$10(4)$

$<0.001$

$10.8(1-38)$

$<0.001$

DCS : day-case surgery

BMI : body mass index 
OSA : obstructive sleep apnea 
Table 2: Cost of surgery and rehospitalization depending on the type of management in Euros.

DCS group $\mathrm{n}=250$

\section{Control group} $\mathrm{n}=250$

\section{Cost of initial surgery}

Mean cost of SG, excluding unplanned admission (range)

Mean cost of SG, including unplanned admission (range)

Mean cost of SG, including cost of admission the day before surgery (range)
2944

(1523-3807)

4195

(1523-8495)

-
5151

(3175-5486)

5151

(3175-5486) 6559

(4585-6896)
$<0.001$

$\mathbf{0 . 0 3}$

$<0.001$

\section{Rehospitalization}

Mean cost of rehospitalization (range)

Median cost of GL (range)

7314

(2570-10090)

7950

(2756-10090)
7559

(2144-16137) $\quad 0.86$

8630

(7696-16137)

0.41

\section{Mean overall cost per patient*}

Excluding unplanned overnight admission and rehospitalization (range)

Including unplanned overnight admission and excluding rehospitalization (range)

Including cost of admission the day before surgery and excluding rehospitalization (range)

Excluding unplanned overnight admission and including rehospitalization (range) Including unplanned overnight admission and rehospitalization (range)

Including cost of admission the day before surgery and including rehospitalization(range)

$$
2988
$$

(1523-3807)

3294

(1523-8495)

(1523-8495)

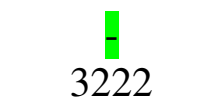

(1523-11621)

3519

(1523-11621)

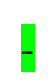

5149

(3175-5486)

5149

$(3175-5486) \quad<0.001$

6559

(4585-6896) <0.001

$(3175-19332) \quad<0.001$

5453

$(3175-19332) \quad<0.001$

6863

$(4585-20742) \quad<\mathbf{0 . 0 0 1}$

\section{Overall cost $* *$}

Excluding unplanned overnight admission and rehospitalization (range)

Including unplanned overnight admission and excluding rehospitalization (range) Including cost of admission the day before surgery and excluding rehospitalization

$\begin{array}{rrr}644694 & 1235766 & <\mathbf{0 . 0 0 1} \\ 773616 & 1235766 & <\mathbf{0 . 0 0 1} \\ & 1588266 & <\mathbf{0 . 0 0 1}\end{array}$




\section{(range)}

Excluding unplanned overnight admission

and including rehospitalization (range)

Including unplanned overnight admission

and rehospitalization (range)

741281

$1363341<\mathbf{0 . 0 0 1}$

Including cost of admission the day before

879800

1363341

$<0.001$

surgery and including

rehospitalization(range)

* Mean overall cost per patient represents the mean cost per patient, including the cost of the hospital stay during primary SG and the cost of rehospitalization in the case of postoperative complications during the first 3 postoperative months.

** Overall cost represents the cost of the hospital stay during primary SG and the cost of rehospitalization in the case of postoperative complications during the first 3 postoperative months for all 250 patients.

DCS: day-case surgery

SG: sleeve gastrectomy

GL: gastric leak 
Table 3: Changing modalities of SG as DCS over time

First 100 cases Subsequent 150

[Rebibo]

cases

\section{Preoperative data}

Age

(range)

Female gender (\%)

BMI (range)

$\mathrm{BMI} \geq 50 \mathrm{~kg} / \mathrm{m}^{2}(\%)$

Type 2 Diabetes (\%)

Hypertension (\%)

Dyslipidemia (\%)

Metabolic syndrome (\%)

OSA $(\%)$

$\begin{array}{ccc}35.9(22-55) & 35(18-58) & 0.84 \\ 93(93) & 139(92.6) & 0.95 \\ 42.4(37.5-50.8) & 42(35-52.3) & 0.87 \\ 2(2) & 8(5.3) & 0.49 \\ 6(6) & 4(2.6) & 0.35 \\ 13(13) & 30(20) & 0.31 \\ 7(7) & 16(10.6) & 0.39 \\ 2(2) & 5(2) & 0.90 \\ 0(0) & 6(4) & 0.04\end{array}$

\section{Postoperative data}

Unplanned overnight admission (\%)

$8(8)$

$11(7.3)$

0.97

Postoperative mortality (\%)

$0(0)$

$0(0)$

$9(9)$

$5(3.3)$

0.005

Major postoperative complications (\%)

5 (5)

$1(0.6)$

0.03

Reoperation (\%)

$3(1.2)$

$0(0)$

0.03

Type of complications

Gastric leak (\%)

Postoperative bleeding (\%)

$3(3)$

$1(0.6)$

0.01

Postoperative hematoma (\%)

$0(0)$

$0(0)$

$1(1)$

$1(0.6)$

0.08

Gastric stenosis (\%)

$1(0.4)$

$0(0)$

0.22

Unscheduled consultation (\%)

7 (7)

$9(6)$

0.10

Rehospitalization (\%)

7 (7)

7 (4.6)

0.03

DCS : day-case surgery

SG : sleeve gastrectomy

BMI : body mass index

OSA : obstructive sleep apnea 